Original Article

\title{
Effects of virtual reality training with modified constraint-induced movement therapy on upper extremity function in acute stage stroke: a preliminary study
}

\author{
Eun-Kyu Ji, OT, MSc ${ }^{1)}$, SANG-HeOn LeE, OT, $\left.\mathrm{PhD}^{2}\right)^{*}$ \\ 1) St. Vincent's Hospital, Republic of Korea \\ 2) Department of Medical Science, College of Medical Science, Soonchunhyang University: \\ 22 Soonchunhyang-ro, Sinchang-myeon, Asan-si, Chungcheongnamd-do 31538, Republic of Korea
}

\begin{abstract}
Purpose] The purpose of this study was to investigate the effects of virtual reality training combined with modified constraint-induced movement therapy on upper extremity motor function recovery in acute stage stroke patients. [Subjects and Methods] Four acute stage stroke patients participated in the study. A multiple baseline single subject experimental design was utilized. Modified constraint-induced movement therapy was used according to the EXplaining PLastICITy after stroke protocol during baseline sessions. Virtual reality training with modified constraint-induced movement therapy was applied during treatment sessions. The Manual Function Test and the Box and Block Test were used to measure upper extremity function before every session. [Results] The subjects' upper extremity function improved during the intervention period. [Conclusion] Virtual reality training combined with modified constraint-induced movement is effective for upper extremity function recovery in acute stroke patients.

Key words: Virtual reality training, Upper extremity function, Modified constraint-induced movement therapy
\end{abstract}

(This article was submitted Jun. 20, 2016, and was accepted Jul. 27, 2016)

\section{INTRODUCTION}

Stroke, malignant neoplasms, and heart disease are the leading causes of death in the Republic of Korea. Stroke survivors can have serious disabilities including reduced motor, sensory, and language function. About $75 \%$ of acute stroke patients exhibit upper extremity paralysis. After stroke, muscle weakness, abnormal muscle tone, and range of motion limitation occur restricting the use of the upper extremity. Independent activities of daily living and the quality of life decrease.

In a cohort study of 1,716 stroke patients, functional recoveries after 6 months were better in patients who started intervention within 7 days than in those who started within 30 days $^{1,2)}$. In an animal study about neuroplasticity after stroke, the highest reaction seem to occur at a time point between 3 and 4 weeks after stroke. If motor intervention can begin as soon as possible, functional recovery can be improved and disability can be minimized ${ }^{3-5)}$.

Several approaches exist for upper extremity motor function rehabilitation after stroke including motor function recovery bilateral training, constraint-induced movement therapy (CIMT), modified constraint-induced movement therapy (m-CIMT), electrical stimulation, high-intensity therapy, repetitive task training, robotic therapy, and virtual reality training (VRT) ${ }^{6-9)}$.

In the Korean standard medical treatment guide, patients with active wrist extension, thumb abduction, active extension of more than 10 degrees in two fingers and sufficient balance skills are recommended to undergo CIMT level A, 1++ ${ }^{10)}$. However, patients undergoing CIMT can experience emotional anxiety and pressure due to the intensive treatment and the restraint of the less affected side. In a meta-analysis on the treatment effects of CIMT, the emotional factor influence treatment 
results $^{11)}$. Patients receiving intensive CIMT frequently became frustrated, hindering their improvement ${ }^{12)}$. Another treatment method to improve upper extremity function is VRT. VRT involves a human-computer interface, allowing people to feel that they are really interacting with the environment even though it is computerized. VRT can result in brain re-organization. Recently, therapeutic VRT has been widely developed and applied in clinical trials in the rehabilitation field ${ }^{13)}$. One of the main differences between VRT and other treatment approaches is to use fun and motivational video games to improve upper extremity and hand function ${ }^{13}$.

Past studies have shown that m-CIMT had positive results in the subacute and chronic phases of stroke patients of upper extremity function, but it was less effective in the acute phase. VRT was shown to improve upper extremity function. Flynn et al. showed in 2007 that applying video games to subacute stroke patients improved upper extremity function ${ }^{14)}$. In the evidence-based review of stroke rehabilitation, chronic stroke patients responded positively with respect to upper extremity rehabilitation after VRT. To date, no study has been conducted, to our knowledge, about the combined effects of VRT and $\mathrm{m}$-CIMT in acute stage stroke patients. The purpose of this study is to determine the combined effects of VRT and $\mathrm{m}$-CIMT on acute phase hemiplegic stroke patients' upper extremity function.

\section{SUBJECTS AND METHODS}

This preliminary study included 4 people to determine the potential of combining VRT and m-CIMT for recovery of upper extremity function after stroke. Prior to initiating the study, ethical approval was granted from the Institutional Review Board on Human Subjects Research and Ethics Committees, Soonchunhyang University, Cheonan, Korea. Patients signed informed consent.

The 4 participants were in the acute stages of stroke with hemiparesis and hospitalized at St. Vincent's Hospital in the Republic of Korea between February 2015 and May 2015. All individuals participated voluntarily.

Stroke patients who:

1) Were diagnosed with stroke by a doctor

2) Were admitted to inpatient/outpatient rehabilitation within 3 months of stroke onset.

3) Could actively extend the wrist of the hemiparetic limb at least 20 degree and metacarpophalangeal joints at least 10 degree.

4) Had a score higher than 24 points on the Korean mini-mental state examination (K-MMSE)

5) Had higher than Grade 2 for manual muscle testing (MMT)

6) Had Berg Balance Test (BBS) scores higher than 40 points were included.

Subjects with visual hemineglect, hemianopsia, visual-perceptual dysfunction, sensory deficits, seizure, hypertension, or angina pectoris were excluded from the study.

Case 1 was that of a 70-year-old male who exhibited left hemiplegia due to a right middle cerebral artery infarction and had a K-MMSE score of 28 and a Motor-Free Visual Perception Test (MVPT) score of 26. Case 2 was that of a 74-year-old male who exhibited right hemiplegia due to a left pontine infarction and had a K-MMSE score of 30 and a MVPT score of 28. Case 3 was a 52-year-old male who exhibited right hemiplegia due to a left pontine infarction and had a K-MMSE score of 26 and a MVPT score of 33. Case 4 was a 35-year-old male who exhibited left hemiplegia due to a right basal ganglia intracerebral hemorrhage and had a K-MMSE score of 28 and a MVPT score of 31. General characteristics of these subjects are given in Table 1.

The research design of this preliminary study was a single subject experimental AB design with multiple baselines. The study was conducted 5 weeks from February to March 2015 after IRB approval. m-CIMT using the EXplaining PLastICITy after stroke (EXPLICIT-stroke) - m-CIMT protocol was applied during the baseline period for 5 sessions/ 1 week ( 30 minutes per session). m-CIMT with VRT was applied during the intervention period for 15 sessions/4 weeks (30 minutes per session). The Manual Function Test (MFT) and the Box and Block Test (BBT) were conducted before the second and fourth session of the 5 sessions/ 1 week course.

$\mathrm{m}$-CIMT has been used on the basis of the study by Page et al ${ }^{15)}$. Subjects were received for 5 times per week and each intensive treatment time was 30 minutes using the EXPLICIT-stroke m-CIMT protocol. This protocol utilizes a bottom-up approach consisting of 3 stages. The first stage is for training gross movement. The second stage is for grasping and gripping. The last stage is for in-hand manipulation. The unaffected upper extremity should be limited for 5 hours a day. A checklist was used to allow the caregiver to confirm that this protocol was followed.

Table 1. General characteristics of subjects

\begin{tabular}{lcllcccc}
\hline & Gender & Diagnosis & Affected side & Onset time & Age (years) & K-MMSE & MVPT \\
\hline CASE 1 & Male & Rt. MCA infarction & Lt. hemiplegia & 4 weeks & 70 & 28 & 26 \\
CASE 2 & Male & Lt. Pontine infarction & Rt. hemiplegia & 3 weeks & 74 & 30 & 28 \\
CASE 3 & Male & Lt. Pontine infarction & Rt. hemiplegia & 1 week & 52 & 26 & 33 \\
CASE 4 & Male & Rt. BG ICH & Lt. hemiplegia & 5 weeks & 35 & 28 & 31 \\
\hline
\end{tabular}


The VRT protocol included using Microsoft Xbox Kinect Adventures software that works the upper extremities such as 'Rallyball', '20,000 Leaks', and 'Reflex Ridge'. The affected upper extremity was utilized for 10 minutes per program at C hospital under quiet circumstances.

In the 'Rallyball' game, balls approaching the player from multiple angles are hit from a chair facing a screen. Each game takes 3 minutes and the game was played 3 times during the study by the participant. The ' 20,000 Leaks' game is played by blocking leaky holes using a hand no a screen. Each game takes 3 minutes and the game was played 5 times during the study. The 'Reflex Ridge' game involves hitting, grasping obstacles and moving while sitting in a chair. Each game takes 2 minutes and the game was played 5 times during the study.

BBT, a test of manual dexterity, has been used by occupational therapists and others to evaluate physically handicapped individuals ${ }^{16}$. Required materials are one wooden box, 150 cubes $(2.5 \mathrm{~cm})$, a stopwatch, and an evaluation form. Patients first use the less affected side while sitting and passing cubes during a limited time period. The patient is allowed a 15-second trial period prior to testing. When testing begins, the patient should grasp one block at a time with the dominant hand, transport the block over the partition, and release it into the opposite compartment. The patient should continue doing this for one minute. The examiner count the released blocks.

MFT has been used for estimating and recording changes in upper extremity motor function to monitor recovery progress during recovery after stroke. MFT is composed of 32 test items, which examine arm motions and manipulative activities. It takes 10 minutes per test. The test was conducted when sitting on a chair with a back under quiet circumstances. Total score is 18 .

MFT and BBT mean during baseline and treatment sessions, and raw scores for every second and fourth sessions of a week were compared to determine functional recovery of each upper extremity.

\section{RESULTS}

BBT mean score was improved after treatment. In Case 1, the BBT pretest average was 19 and the posttest average was 21.55. In Case 2, the pretest average was 41 and the posttest average was 50.42. In Case 3, the pretest average was 7.16 and the posttest average was 12.8. In Case 4, the pretest average was 28.5 and the posttest average was 32.66 (Tables 2, 3).

For result analysis, The MFT mean score was also improved after treatment. In Case 1, the MFT pretest average was 6 and the posttest average was 6.55. In Case 2, the pretest average was 11.75 and the posttest average was 12.57 . In Case 3, the pretest average was 3.83 and the posttest average was 5.6. In Case 4, the pretest average was 8.62 and the posttest average was 9.00 (Tables 2,4$)$.

Table 2. Comparisons of the average score before and after intervention in BBT and MFT

\begin{tabular}{lrrrr}
\hline & \multicolumn{2}{c}{ Box and Block Test (number) } & \multicolumn{2}{c}{ Manual Function Test (score) } \\
\cline { 2 - 5 } & \multicolumn{1}{c}{ Pretest } & Posttest & Pretest & \multicolumn{1}{c}{ Posttest } \\
\hline Case 1 & $19.00 \pm 0.00$ & $21.55 \pm 2.85$ & $6.00 \pm 0.00$ & $6.55 \pm 0.73$ \\
Case 2 & $41.00 \pm 0.71$ & $50.42 \pm 2.70$ & $11.75 \pm 0.58$ & $12.57 \pm 0.53$ \\
Case 3 & $7.16 \pm 2.39$ & $12.80 \pm 1.92$ & $3.83 \pm 0.89$ & $5.60 \pm 0.55$ \\
Case 4 & $28.5 \pm 0.69$ & $32.66 \pm 2.52$ & $8.62 \pm 0.76$ & $9.00 \pm 1.00$ \\
\hline
\end{tabular}

Table 3. Box and Block Test scores

\begin{tabular}{lcccccccccc}
\hline & Session 1 & Session 2 & Session 3 & Session 4 & Session 5 & Session 6 & Session 7 & Session 8 & Session 9 & Session 10 \\
\hline Case 1 & 21 & $17 *$ & 26 & 24 & 19 & 21 & 23 & 21 & 18 & 25 \\
Case 2 & 36 & 37 & 43 & $48^{*}$ & 51 & 55 & 47 & 51 & 52 & 49 \\
Case 3 & 3 & 3 & 9 & 6 & 8 & $12^{*}$ & 14 & 15 & 13 & 10 \\
Case 4 & 27 & 27 & 28 & 27 & 28 & 28 & 28 & $33^{*}$ & 35 & 30 \\
\hline
\end{tabular}

(value $=$ number)

*Intervention start point

Table 4. Manual Function Test scores

\begin{tabular}{lcccccccccc}
\hline & Session 1 & Session 2 & Session3 & Session 4 & Session 5 & Session 6 & Session 7 & Session 8 & Session 9 & Session 10 \\
\hline Case 1 & 6 & $6^{*}$ & 6 & 6 & 6 & 7 & 7 & 7 & 6 & 8 \\
Case 2 & 12 & 12 & 11 & $12^{*}$ & 12 & 12 & 13 & 13 & 13 & 13 \\
Case 3 & 4 & 3 & 3 & 3 & 5 & $5^{*}$ & 5 & 6 & 6 & 6 \\
Case 4 & 10 & 8 & 8 & 9 & 9 & 8 & 9 & $8^{*}$ & 9 & 10 \\
\hline
\end{tabular}

(value $=$ score)

*Intervention start point 


\section{DISCUSSION}

CIMT is a commonly used rehabilitation intervention to improve upper limb function after stroke. CIMT was originally developed for patients with a chronic upper limb paresis. The affected motor cortex was extended after CIMT intervention but restricting the use of the unaffected hand through use of gloves for more than 6 hours leads to discomfort and trouble in the patients ${ }^{17}$. Thus the m-CIMT protocol was developed. To preserve and enhance the effects of m-CIMT on the function of the upper extremities, the necessity of using both hands during daily activities is emphasized and encouraged.

VRT has been used effectively for upper extremity rehabilitation, especially in fearful subjects. There are two types of inexpensive, commonly available gaming systems that can be integrated into rehabilitation programs: controller-based and camera tracking-based. The camera tracking device can prevent compensatory movement and the subject does not need to hold the controller ${ }^{17)}$. The neurological background for use of VRT is the mirror neuron theory. When the pre-motor cortex was observed in monkeys using VRT, the mirror neuron was activated ${ }^{18)}$. This training also resulted in brain re-organization. After VRT, both primary sensorimotor cortexes, the unaffected side premotor cortex and the affected or unaffected supplementary motor area were activated, and motor function was improved ${ }^{17}$. Also, Holden et al. said that VRT can be enjoyable since it is done through a variety of gaming media ${ }^{19}$. The purpose of this study was to identify the combined effect of VRT and m-CIMT for upper extremity function recovery.

The kinetic system used in this study utilized camera tracking, which has the advantage of preventing compensatory movement. Nintendo Wii and PlayStation are both based on controllers. Patients had difficulty realizing when another person or object entered into range of the camera. When using the kinetic system, patients must maintain their own balance in the sitting position. There have been previous studies about the relationship between balance and gait capacity after combined intervention with VRT and m-CIMT. When treating stroke patients with VRT and m-CIMT using the Wii fit game, the balance capacity improved ${ }^{20-22)}$.

Upper extremity function improved in all 4 cases included in this study. Thus, the combination of VRT and m-CIMT can be applied in upper extremity rehabilitation after stroke. In the future studies, it need to investigate the effects of VRT on the participation level with an adequate follow-up period. The number of participants should increase in order to be able to generalize the effects of this combined therapy and compare the combination of VRT with m-CIMT against each type of therapy used individually. Quality of life should be considered when evaluating the effects of VRT with m-CIMT on activities of daily living.

\section{ACKNOWLEDGEMENT}

This work was supported by the Soonchunhyang University Research Fund.

\section{REFERENCES}

1) Paolucci S, Antonucci G, Grasso MG, et al.: Early versus delayed inpatient stroke rehabilitation: a matched comparison conducted in Italy. Arch Phys Med Rehabil, 2000, 81: 695-700. [Medline] [CrossRef]

2) Musicco M, Emberti L, Nappi G, et al., Italian Multicenter Study on Outcomes of Rehabilitation of Neurological Patients: Early and long-term outcome of rehabilitation in stroke patients: the role of patient characteristics, time of initiation, and duration of interventions. Arch Phys Med Rehabil, 2003, 84: 551-558. [Medline] [CrossRef]

3) Ottenbacher KJ, Jannell S: The results of clinical trials in stroke rehabilitation research. Arch Neurol, 1993, 50: 37-44. [Medline] [CrossRef]

4) Cifu DX, Stewart DG: Factors affecting functional outcome after stroke: a critical review of rehabilitation interventions. Arch Phys Med Rehabil, 1999, 80: S35-S39. [Medline] [CrossRef]

5) Kwakkel G, Kollen BJ, van der Grond J, et al.: Probability of regaining dexterity in the flaccid upper limb: impact of severity of paresis and time since onset in acute stroke. Stroke, 2003, 34: 2181-2186. [Medline] [CrossRef]

6) Laver K, George S, Thomas S, et al.: Cochrane review: virtual reality for stroke rehabilitation. Eur J Phys Rehabil Med, 2012, 48: 523-530. [Medline]

7) Langhorne P, Bernhardt J, Kwakkel G: Stroke rehabilitation. Lancet, 2011, 377: 1693-1702. [Medline] [CrossRef]

8) Liepert J: Evidence-based therapies for upper extremity dysfunction. Curr Opin Neurol, 2010, 23: 678-682. [Medline] [CrossRef]

9) Oujamaa L, Relave I, Froger J, et al.: Rehabilitation of arm function after stroke. Literature review. Ann Phys Rehabil Med, 2009, 52: 269-293. [Medline] [CrossRef]

10) Rah UW, Kim YH, Ohn SH, et al.: Clinical practice guideline for stoke rehabilitation in Korea 2012. Brain Neurorehabil, 2014, 7. [CrossRef]

11) Sin H, Lee G: Additional virtual reality training using Xbox Kinect in stroke survivors with hemiplegia. Am J Phys Med Rehabil, 2013, 92: 871-880. [Medline] [CrossRef]

12) Dromerick AW, Edwards DF, Hahn M: Does the application of constraint-induced movement therapy during acute rehabilitation reduce arm impairment after ischemic stroke? Stroke, 2000, 31: 2984-2988. [Medline] [CrossRef]

13) Saposnik G, Levin M, Outcome Research Canada (SORCan) Working Group: Virtual reality in stroke rehabilitation: a meta-analysis and implications for clinicians. Stroke, 2011, 42: 1380-1386. [Medline] [CrossRef] 
14) Flynn S, Palma P, Bender A: Feasibility of using the Sony PlayStation 2 gaming platform for an individual poststroke: a case report. J Neurol Phys Ther, 2007, 31: 180-189. [Medline] [CrossRef]

15) Page SJ, Levine P, Leonard A, et al.: Modified constraint-induced therapy in chronic stroke: results of a single-blinded randomized controlled trial. Phys Ther, 2008, 88: 333-340. [Medline] [CrossRef]

16) Mathiowetz V, Volland G, Kashman N, et al.: Adult norms for the Box and Block Test of manual dexterity. Am J Occup Ther, 1985, 39: 386-391. [Medline] [CrossRef]

17) Rizzolatti G, Fadiga L, Fogassi L, et al.: Resonance behaviors and mirror neurons. Arch Ital Biol, 1999, 137: 85-100. [Medline]

18) Anderson KR, Woodbury ML, Phillips K, et al.: Virtual reality video games to promote movement recovery in stroke rehabilitation: a guide for clinicians. Arch Phys Med Rehabil, 2015, 96: 973-976. [Medline] [CrossRef]

19) Jang SH, You SH, Hallett M, et al.: Cortical reorganization and associated functional motor recovery after virtual reality in patients with chronic stroke: an experimenter-blind preliminary study. Arch Phys Med Rehabil, 2005, 86: 2218-2223. [Medline] [CrossRef]

20) Holden MK, Dyar T: Virtual environment training: a new tool for neurorehabilitation. J Neurol Phys Ther, 2002, $26: 62-74$.

21) Fluet GG, Deutsch JE: Virtual reality for sensorimotor rehabilitation post-stroke: the promise and current state of the field. Curr Phys Med Rehabil Rep, 2013, 1: 9-20. [Medline] [CrossRef]

22) Lohse KR, Hilderman CGE, Cheung KL, et al.: Virtual reality therapy for adults post-stroke: a systematic review and meta-analysis exploring virtual environments and commercial games in therapy. PLoS One, 2014, 9 doi: 10.1371/journal.pone.0093318. 